\title{
Brock, Lothar, und Hendrik Simon (Hrsg.) (2021): The Justification of War and International Order. From Past to Present
}

\author{
Oxford: Oxford University Press. 560 Seiten. $\$ 95.00$
}

\section{Theresa Reinold}

Angenommen: 22. Dezember 2021 / Online publiziert: 11. Januar 2022

(C) Der/die Autor(en) 2022

Der von Lothar Brock und Hendrik Simon herausgegebene Sammelband „, The Justification of War and International Order. From Past to Present" zeichnet detailliert die Rechtfertigungsdiskurse von Belligerenten über die Jahrhunderte nach, um dadurch die zentrale These des Buchs zu untermauern, der zufolge die Geschichte des Krieges auch eine Geschichte seiner Rechtfertigung sei. Für dieses Unterfangen bringen die Herausgeber eine Reihe von Wissenschaftlerinnen und Wissenschaftlern aus verschiedenen Disziplinen - der Rechtswissenschaft, Soziologie, Geschichtswissenschaft sowie den Internationalen Beziehungen (IB) - zusammen, die in verschiedenen Kapiteln die Kriegsrechtfertigungsdiskurse von Kriegsparteien in diversen historischen Epochen beleuchten.

Der Band gliedert sich, Einleitung und Conclusio einmal ausgenommen, in sieben Teile: Teil I präsentiert (allerdings recht kurz und bündig) grundlegende theoretische Überlegungen zum Thema Krieg und Ordnung(sbildung). Im zweiten Teil des Bandes werden die Rechtfertigungsdiskurse der Frühmoderne in den Blick genommen, gefolgt von einer Analyse der Kriegsdiskurse im 19. Jahrhundert (Teil III). Der vierte Teil befasst sich mit der Rhetorik von Belligerenten in der ersten Hälfte des 19. Jahrhunderts. Dem schließen sich eine Reihe von Beiträgen an, die die Argumentationsmuster der kriegsführenden Parteien im Globalen Norden und Süden in der zweiten Hälfte des 20. Jahrhunderts und dem frühen 21. Jahrhundert untersuchen (Teile V-VII) und verschiedene Formen der Intervention - von Militäroperationen im Kampf gegen den Terrorismus über prodemokratische Intervention bis hin zu Interventionen zum Schutz von Zivilisten - thematisieren.

Was nun die Stärken und Schwächen des vorliegenden Werkes angeht, so ist in jedem Falle die interdisziplinäre Ausrichtung des Bandes positiv hervorzuheben,

Theresa Reinold $(\varangle)$

Institut für Politikwissenschaft, Universität Duisburg-Essen, Duisburg, Deutschland

E-Mail: theresa.reinold@uni-due.de 
der Stimmen aus verschiedenen Fachrichtungen hörbar macht und sich explizit zum Ziel gesetzt hat, Autorinnen und Autoren aus dem Globalen Norden und dem Globalen Süden miteinander ins Gespräch zu bringen. Dies ist ein durchaus wichtiger Beitrag zur Überwindung der Hegemonie des Globalen Nordens, die nach wie vor akademische Debatten im Bereich der zwischenstaatlichen Gewaltanwendung prägt (ebenso wie diese Gewaltanwendung selbst). In diesem Zusammenhang ist auch der Beitrag, welchen der Band zur Erforschung des Themas „mestizo international law“ leistet (S. 17 und in den Kapiteln von Siddharth Mallavarapu sowie B. S. Chimni), erwähnenswert und verweist auf ein Forschungsgebiet, dem zukünftig noch mehr Aufmerksamkeit gewidmet werden sollte.

Demgegenüber erscheint allerdings das Ausgangsargument des Bandes - dass die Geschichte des Krieges auch die Geschichte seiner Begründung sei - nicht wirklich kontraintuitiv und innovativ, weder im Bereich der IB noch im Völkerrecht. Auch hart gesottene IB-Realisten würden dem nicht widersprechen und Konstruktivistinnen mit ihrer Betonung des Akteur-Struktur Nexus' schon gar nicht - so ist eine der Grundannahmen der konstruktivistischen Schule in den IB, dass Diskurse (und Praktiken) internationale Strukturen bzw. normative Ordnungen erzeugen (oder aber auch destabilisieren und gegebenenfalls transformieren). Doch auch Anhänger der realistischen Theorie der IB könnten sich problemlos der Kernthese der Herausgeber, wie sie auf S. 4 der Einleitung artikuliert wird, anschließen: „Our fundamental thesis is that in their justifications of war, states and other political actors refer to (existing or presumed) norms of the international order to depict their own violence as legitimate, that is ,appropriate behavior". "Natürlich tun sie dies - aus realistischer Sicht vor allem deswegen, weil der Anschein von Legitimität bzw. Legalität möglichen Widerstand anderer Staaten gegen die Politik des Hegemonen reduziert. Dies hat bereits der Mitbegründer der realistischen Schule Hans Morgenthau in seinem Werk „In Defense of the National Interest“ anerkannt, indem er den Missbrauch universalistischer Rhetorik als Feigenblatt für Großmachtimperalismus beschreibt. Kriegsrechtfertigungsdiskurse tragen also nicht zwangsweise zu Ordnungsbildung bei, sondern können ebenso gut Versuche sein, imperiale Erschütterungen eben dieser Ordnung zu beschönigen. Ja, auch die Putins, Bush Juniors und Trumps dieser Welt berufen sich auf (imaginäre) völkerrechtliche Normen, um ihre Interventionen zu legitimieren, dennoch erscheint es absurd, zu argumentieren, dass beispielsweise die amerikanische Invasion des Irak im Jahr 2003 oder die russische Annexion der Krim im Jahr 2014 zur internationalen Ordnungsbildung beigetragen hätten. Es greift also etwas zu kurz, zu argumentieren, dass allein der rhetorische Verweis auf eine (imaginäre oder real existierende) normative Ordnung einen Zivilisierungsfortschritt darstellt. Die eigentlich interessante Frage ist doch vielmehr, welche Diskurse, welche Rechtfertigungsmuster die existierende Ordnung stabilisieren und welche sich als disruptiv für eben diese Ordnung erweisen.

Insofern liegt die zentrale Schwäche eines ansonsten sehr lesenswerten Sammelbandes in dem zu laxen Umgang mit fundamentalen Konzepten, die für sich genommen nicht ausreichend Tiefenschärfe besitzen und im Verhältnis zueinander nicht genügend differenziert werden. Wie beispielsweise verhält sich das Konzept der Ordnungsbildung zu dem Konzept der Rule of Law bzw. „legalization“ - fundamentale Begriffe, die der Kernfrage des Sammelbandes zugrunde liegen, aber nicht 
ausreichend reflektiert werden. Zwar verweisen die Herausgeber wiederholt auf das Konzept der Rule of Law (beispielsweise auf S. 518-519), setzen sich aber nicht detaillierter mit der Bedeutung des Begriffs auseinander und erklären ebenso wenig, wie es sich zu ihrem Verständnis von Ordnung verhält. Damit zusammen hängt auch die Frage, wie man qualitativ zwischen verschiedenen normativen Ordnungen, wie sie laut Brock und Simon aus Rechtfertigungsdiskursen hervorgehen, unterscheiden kann. Die Herausgeber verhalten sich zu dieser Frage nicht wirklich. So schreiben sie auf S. $4,[\ldots]$ international order is to be understood as a ,normative order" in which norms function as an instrument of politics (including the politics of theorybuilding) and at the same time structure the practice of justifying and practicing the use of force". Auf S. 13-14 setzen sich die Autoren noch einmal mit dem Ordnungsbegriff auseinander, allerdings auch eher kursorisch, beschränkt auf wenige Absätze. Hier kommen sie wenig überraschend zu dem Schluss, dass die internationale Ordnung durch Macht ebenso wie durch Normen konstituiert werde (S. 14). Es erscheint mir hingegen sinnvoll, über derart allgemeine Aussagen hinauszugehen und die Qualität einer normativen Ordnung vielmehr danach zu bewerten, inwiefern sie den Spielraum für die Ausübung politischer Macht und Willkür einschränkt. Diese Definition von Ordnungsbildung würde dann den Kern der Idee der Rule of Law widerspiegeln und somit zwei zentrale Konzepte des Sammelbandes besser zueinander in Beziehung setzen.

In der Conclusio reflektieren die Herausgeber noch einmal die wichtigsten Erkenntnisse des Bandes und kommen zu dem Schluss, dass ,the complex discourses on the justification of war analyzed in this book confirm that justifications of war are more than ,cheap talk' without normative meaning. Even propaganda refers to an audience which constitutes itself around certain normative expectations. Whether such propaganda works is dependent on what a larger audience regards as acceptable“ (S. 523). Was aber diese ,larger audience“ oder ,interpretive community“, wie sie Stanley Fish beschrieben hat, als akzeptabel betrachtet, hängt davon ab, inwiefern der Rechtfertigungsdiskurs der intervenierenden Partei bzw. Parteien eine gewisse Kohärenz mit existierenden, kollektiv geteilten Standards angemessenen Verhaltens aufweist. Kann eine solche Kohärenz nicht hergestellt werden, so trägt die Interventionsrhetorik nicht etwa zur Ordnungsbildung bei, sondern stellt einen (erfolglosen) Versuch dar, eben diese Ordnung zu untergraben.

Funding Open Access funding enabled and organized by Projekt DEAL.

Open Access Dieser Artikel wird unter der Creative Commons Namensnennung 4.0 International Lizenz veröffentlicht, welche die Nutzung, Vervielfältigung, Bearbeitung, Verbreitung und Wiedergabe in jeglichem Medium und Format erlaubt, sofern Sie den/die ursprünglichen Autor(en) und die Quelle ordnungsgemäß nennen, einen Link zur Creative Commons Lizenz beifügen und angeben, ob Änderungen vorgenommen wurden.

Die in diesem Artikel enthaltenen Bilder und sonstiges Drittmaterial unterliegen ebenfalls der genannten Creative Commons Lizenz, sofern sich aus der Abbildungslegende nichts anderes ergibt. Sofern das betreffende Material nicht unter der genannten Creative Commons Lizenz steht und die betreffende Handlung nicht nach gesetzlichen Vorschriften erlaubt ist, ist für die oben aufgeführten Weiterverwendungen des Materials die Einwilligung des jeweiligen Rechteinhabers einzuholen. 
Weitere Details zur Lizenz entnehmen Sie bitte der Lizenzinformation auf http://creativecommons.org/ licenses/by/4.0/deed.de. 